\title{
Rendimiento de habla espontánea y canto en usuarios con accidente cerebro vascular cerebeloso: un estudio de caso
}

\author{
María Soledad Sandoval Zúñiga'; Carolina Henríquez Espejo²; Liza Henríquez Navarrete²; Andrea Elis- \\ salde Reyes ${ }^{2}$; Diego Rodríguez Rivas ${ }^{2}$
}

Recibido 9 de mayo de 2019 / Primera revisión 8 de agosto de 2019 / Aceptado 17 de noviembre de 2019

Resumen. El propósito de este estudio fue caracterizar los rendimientos en habla y en canto de dos pacientes con disartria atáxica a causa de un accidente cerebro vascular (ACV) cerebeloso con el fin de describir sus resultados en relación a las características clínicas y sociodemográficas que poseen.

Corresponde a un estudio de caso de tipo cuantitativo, con un diseño no experimental-transversal y un alcance descriptivo. Ambos sufrieron un ACV cerebeloso pero difieren en las características de la disartria, la técnica vocal, la edad, la frecuencia terapéutica, entre otras. Los instrumentos que se utilizaron para la medición de las variables fueron: el Protocolo de Evaluación de Habla (González y Toledo, 2002), el Protocolo de Habla versus Canto para pacientes disártricos, el cuestionario Voice Handicap Index (VHI) de Núñez-Batalla et al. (2007) y la escala RASATI (Pinho y Pontes, 2002).

Los resultados indicaron que quien padece de una disartria atáxica leve presenta mejores resultados en habla y en canto en comparación con aquel que padece de una disartria moderada. Esto se explica por las diversas diferencias que ambos poseen en cuanto a la severidad del cuadro, a las características neurológicas, a la frecuencia de terapia, a la edad y a la técnica vocal previa al evento vascular.

Ambos sufren alteraciones en los procesos motores básicos de respiración, fonación y prosodia.

Palabras Claves: Habla; canto; disartria atáxica; técnica vocal; ACV cerebeloso.

\section{[en] Speech and singing performance in patients suffering a cerebellar stroke: a case study}

Abstract. The objective of this study was to characterize speech and singing performances of two subjects who suffered ataxic dysarthria due to a cerebellar stroke for describing their results according their clinical and sociodemographic characteristics.

This study was of quantitative type, with a non-experimental-transversal design with a descriptive scope. Both patients suffered a cerebellar stroke but they differed in the characteristics of dysarthria, vocal technique, age, and rehabilitation frequency, between others factors. The instruments that were used to measure varibales corresponding to: Protocolo de Evaluación de Habla (González y Toledo, 2002), Protocolo de Habla versus Canto para pacientes disártricos, the Voice Handicap Index (VHI) by Núñez-Batalla et al. (2007) and the RASATI scale (Pinho and Pontes, 2002).

The results indicated that who suffers a mild dysarthria has better speech and singing results compared to the subject who suffers from a moderate dysarthria due to the differences that they posse like pathology severity, neurological characteristic, therapy frequency, age and vocal technique before the vascular event.

Both subjects showed alterations in basic motor processes in respiration, phonation and prosody.

Key Words: Speech, singing, ataxic dysarthria, vocal technique, cerebellar stroke.

Sumario: 1. Introducción. 2. Metodología. 2.1. Muestra. 2.2. Instrumentos. 2.3. Procedimiento. 3. Resultados. 4. Discusión y conclusiones. 6 . Referencias bibliográficas.

Cómo citar: Sandoval Zúñiga, M. S. et al. (2020) Rendimiento de habla espontánea y canto en usuarios con accidente cerebro vascular cerebeloso: un estudio de caso, Revista de investigación en Logopedia 10(1), 43-51.

\footnotetext{
Universidad Pedro de Valdivia, Chile.

Universidad Adventista de Chile, Chile.

2 Universidad San Sebastián, Chile.

Rev. investig. logop. 10(1) 2020: 43-51
} 


\section{Introducción}

El accidente cerebro vascular (ACV) es una de las patologías neurológicas más frecuentes en Latinoamérica y en el mundo. Según la Organización Mundial de la Salud (OMS), cada año quince millones de personas sufren una lesión vascular, de las cuales cinco millones fallecen y cinco millones quedan secuelados (Ministerio de Salud [MINSAL], 2015).

En Chile, el accidente cerebro vascular es la principal causa de muerte en pacientes adultos y cuenta con 8.736 fallecidos al año, según datos del MINSAL en el año 2011; lo que equivale a un fallecido cada hora por esta causa. Además, es la segunda causa de mortalidad prematura en Chile (MINSAL, 2015). Sin embargo, gracias a los estudios y avances científicos, se ha trabajado en aspectos importantes tales como la promoción en aspectos generales de salud, prevención de la enfermedad y la rehabilitación neurológica, con la finalidad de que las tasas de mortalidad vayan en decadencia. Otros elementos en estudio han sido las limitaciones y las deficiencias posteriores al ACV, consideradas como aspectos secuelares en las que se incluyen, desde el punto de vista logopédico, trastornos de habla, de deglución, de cognición y de lenguaje.

La lesión cerebral, a causa de una patología vascular como la expuesta, puede generarse en diversas localizaciones. Un sitio de lesión cerebral característico de los trastornos del habla ocurre en el cerebelo, estructura cerebral que se encarga de la coordinación de los movimientos voluntarios, el control postural, la marcha, la regulación del tono muscular, aspectos emocionales, funciones cognitivas (Ropper, Brown y Klein, 2016), entre otras. Todas estas funciones son necesarias para generar un proceso comunicativo adecuado, que incluyen aspectos de: cognición, habla y lenguaje. El control cerebeloso expuesto permite hablar de forma adecuada y se altera, por lo general, en lesiones de la porción superior del hemisferio cerebeloso izquierdo (Ferri, 2015).

Cabe considerar que quienes sufren de un ACV cerebeloso presentan una disartria de tipo atáxica, donde el grado de severidad dependerá de la afectación de los procesos motores básicos del habla y aspectos funcionales de la misma, que generalmente tienen relación con las características de la lesión cerebral.

Para Duffy (2005), la disartria es un trastorno neurológico del habla caracterizado por alteraciones en la velocidad, la fuerza, la regularidad, el rango, el tono y la exactitud de los movimientos que son necesarios para un adecuado control de la respiración, fonación, resonancia, articulación y prosodia en la producción del habla. Estas alteraciones van a depender del sitio de la lesión cerebral y pueden afectar de forma variable el proceso comunicativo, incluyendo aspectos de habla y canto.

Por otra parte, la fisiopatología de las disartrias se basa en alteraciones del sistema nervioso central o periférico, que se manifiesta por debilidad, incoordinación, movimientos involuntarios y variable tono muscular. El tono muscular en lesiones cerebelosas se encuentra disminuido (hipotonía), lo cual genera pérdida de fuerza y fatiga al realizar movimientos evidenciados por la manifestación clínica ipsilateral, es decir, son observados en la misma parte del cuerpo en la que se produjo la lesión cerebelosa (Ferri, 2015).

Respecto de la especificidad cerebral, es concluyente que un paciente posea esta clínica posterior a una afectación cerebelosa, ya que se ha evidenciado en estudios con resonancia magnética funcional que la función de realizar movimientos repetitivos de labios y lengua, que permiten la articulación de palabras, permite la activación de áreas cerebelosas (Urban, 2013).

El acto motor de hablar es una de las habilidades necesarias para comunicarse, en conjunto con el lenguaje y las funciones cognitivas como la atención y la memoria, permiten generar un proceso comunicativo adecuado. La música o el entrenamiento musical en este sentido, permite la interacción de estas funciones (Montalvo y Moreira-Vera, 2016). Este tipo de destrezas musicales logran potenciar la densidad del tejido neuronal, lo que se evidencia en la corteza auditiva y motora ya que poseen mayor densidad del tejido nervioso. Esto hace posible la modificación de la estructura cerebral, que es proporcional al tiempo de adiestramiento musical (Custodio y Cano - Campos, 2017).

La acción de cantar o hablar se basa en el funcionamiento de los procesos motores básicos. Según González y Bavilacqua (2012), estos procesos son:

1. Respiración: el aire exhalado permite la vibración de las cuerdas vocales y producción de la voz.

2. Fonación: es el sonido generado por la vibración de las cuerdas vocales.

3. Resonancia: corresponde al proceso de amplificación del tono vocal.

4. Articulación: proceso que tiene como finalidad modificar el sonido producido en la laringe mediante órganos fonoarticulatorios (paladar, lengua, labios, etc.).

5. Prosodia: son los aspectos melódicos del habla, incluyen patrones de acentuación, entonación y ritmo.

Cuando estos procesos motores básicos se ven afectados, se genera una alteración en los componentes funcionales del habla. Entre ellos, la naturalidad con la que se habla, la inteligibilidad, la velocidad y la comprensibilidad del mensaje.

Melle (2008), por su parte, indica que al existir una lesión cerebelosa, ésta afectaría el proceso comunicativo, y añade la presencia de clínica más específica como monotonía, tendencia a prolongar la duración de las sílabas, respiración forzada y audible, presencia de hipotonía muscular (la cual genera movimientos alterantes disimétricos, irregulares, reducidos en tasa y recorrido muscular), presencia de ruptura articulatoria, imprecisiones consonánticas 
y vocálicas, acentuación excesiva y tasa de velocidad de habla disminuida. Ferri (2015), por su parte, sostiene que en ocasiones es posible evidenciar un discurso más enlentecido, con una secuenciación y fraseo inadecuado, pausas y sílabas separadas, lo que se conoce como "habla escándida".

Urban (2013) caracteriza el habla de los pacientes con disartria cerebelosa y menciona que las palabras articuladas son irregulares, la intensidad y el tono de la voz se ven afectados involuntariamente, evidenciando una característica de explosión durante la conversación. También poseen pausas que afectan el proceso comunicativo, excesiva separación de las sílabas que generan la impresión de un habla desconectada, imprecisa y con deficiencias articulatorias evidentes. A este patrón de habla se le agrega una respiración forzada y audible, que permite visualizar a alguien descoordinado y cansado. Estas características respiratorias son denominadas "respiración atáxica" (Kent et al., 2000).

En cuanto a los efectos de la música a nivel cerebral, existen variados estudios que enfatizan los beneficios en aspectos cognitivos, psicoemocionales, prosódicos, articulatorios, entre otros. La música es considerada un tipo de lenguaje que permite comunicar emociones mediante el habla. El cerebro que es entrenado musicalmente experimenta particulares cambios en su anatomía y funcionalidad (Custodio y Cano - Campos, 2017). De acuerdo a Soria - Urios, Duque y García - Moreno (2011), se ha evidenciado que existen diferencias cerebrales en el tamaño del cerebro, por ejemplo, mayor simetría y tamaño de la corteza motora, una mayor conectividad interhemisférica y áreas más desarrolladas a nivel cerebral implicadas con la música entre músicos profesionales y pacientes sin entrenamiento musical. Los autores afirman que la música no sólo puede ser un factor preventivo, sino que también es un tipo de tratamiento actualmente muy utilizado, que consiste principalmente en la aplicación terapéutica de la música en disfunciones motores, sensoriales y cognitivas secundarias a alteraciones neurológicas.

Si bien existen múltiples estudios relacionados con la anatomía y funcionamiento del cerebro, el interés de este estudio es el análisis basado en el daño focal a nivel cerebeloso y su implicancia en el proceso de hablar y cantar, con el fin de realizar un análisis exhaustivo de factores relevantes a la hora de evaluar habla y canto en pacientes con antecedentes de ACV cerebeloso para que éstos sean considerados en el proceso de rehabilitación, sobre todo cuando este diagnóstico posee un pronóstico relativamente positivo (Urban, 2013).

La producción de la música implica que nuestro cerebro se active para realizar movimientos específicos, y esto permite pensar que practicar música diariamente durante años tendría repercusiones cerebrales debido a que este órgano se adapta a nuestra necesidades, tanto en estructura como en función (Soria - Urios, Duque y García - Moreno, 2011).

De esta forma, la presente investigación tiene como propósito describir las diferencias en habla y canto entre dos personas que sufrieron un ACV a nivel cerebeloso que difieren en las características clínicas, los antecedentes personales, el tipo de lesión vascular, el entrenamiento vocal previo, las repercusión en la severidad y características del trastorno de habla.

\section{Metodología}

La presente investigación corresponde a un estudio cuantitativo, de tipo no experimental- transversal, con un alcance descriptivo. La finalidad de este estudio es describir y caracterizar cada caso, considerando que son pacientes con antecedentes clínicos y sociodemográficos muy distintos como edad, sexo, técnica vocal, severidad de la disartria, tiempo de rehabilitación, tipo lesión vascular y etiología.

\subsection{Muestra}

La muestra está constituida por dos casos. El primero de ellos es de sexo masculino, quien sufre de un ACV isquémico, evidenciado por una hipodensidad del hemisferio cerebeloso izquierdo, que comprometió Vermis y superficie anterior, lo que generó una disartria atáxica de grado leve; también poseía técnica vocal por tratarse de un cantautor profesional popular de 51 años con aproximadamente 37 años de trayectoria, reconocido por ser autodidacta sin estudios de canto formal. La segunda paciente, en tanto, es de sexo femenino con 32 años de edad, que padece de una disartria atáxica de grado moderado, secundaria a ACV hemorrágico a nivel de hemisferio cerebeloso izquierdo, y sin conocimiento asociado a la técnica vocal. Ambas disartrias fueron originadas por la misma patología de base (ACV cerebeloso), que difieren en 3 meses desde que sufrieron el evento vascular, debido a que el tiempo de evolución de la patología es de 1 año 9 meses en el primer paciente y 1 año 6 meses en la segunda.

Cabe señalar que ambos participantes recibieron terapia logofoniátrica desde la etapa aguda; sin embargo, existen diferencias en el número de sesiones realizadas: el primer paciente recibió un total de 252 sesiones en 21 meses y la paciente 2 una cantidad de 144 sesiones en 18 meses. Además, en cuanto al tipo de lesión vascular, el paciente de sexo masculino sufrió un ACV cerebeloso isquémico por abuso de sustancias y la segunda paciente padece de uno hemorrágico a causa de una crisis hipertensiva.

\subsection{Instrumentos}

En esta investigación se realizaron cuatro evaluaciones por cada participante. En la primera evaluación se utilizó el Protocolo de Evaluación del Habla de González y Toledo (2002), el cual evalúa cinco procesos motores básicos del 
habla: Fonación, Respiración, Resonancia, Articulación y Prosodia. Este protocolo permite, por un lado, determinar el grado de afectación de cada uno de los procesos por medio de una escala de puntajes y, por otro, establecer el diagnóstico correspondiente.

Posteriormente, se evalúo mediante el Protocolo de Habla versus Canto para Pacientes Disártricos, cuya finalidad es evaluar los procesos motores básicos mencionados anteriormente, pero esta vez con estímulos musicales. La prueba se sometió a juicio de tres expertos, quien solicitaron sólo modificaciones menores. Para la aplicación de este instrumento se requiere de un metrónomo y consta de estímulos de habla cantada con distintas velocidades, en un formato grabado, con el ejemplo vocal de lo que se le solicita realizar al paciente. Los parámetros utilizados en esta evaluación corresponden a:

- Logrado: cuando se logra producir el 100\% de la tarea solicitada.

- Medianamente logrado: en el caso de lograr el 50\% de lo solicitado.

- No logrado: cuando no se ejecuta lo solicitado o se realiza en menor proporción del $50 \%$.

Luego se aplicó el cuestionario Voice Handicap Index (VHI) de Núñez-Batalla et al. (2007), que tiene como finalidad cuantificar la percepción de los pacientes en relación a un trastorno vocal. El cuestionario se divide en aspectos funcionales, físicos y emocionales, cuyas preguntas se puntúan entre 0 a 4 y se categorizan en nunca, casi nunca, a veces, casi siempre y siempre.

Finalmente, se utiliza la escala RASATI (Pinho y Pontes, 2002), que es la adaptación de la escala GRBAS (Hirano, 1981); y es considerada una evaluación subjetiva que incluye aspectos perceptivos acústicos, fisiológicos y psicológicos. La escala RASATI mide la calidad de la voz bajo los parámetros de ronquera (R), aspereza (A), soplosidad (S), astenia (A), tensión (T) e inestabilidad vocal (I), en una graduación de 0 a 3 , cuya interpretación es de normal a severo.

\subsection{Procedimiento}

La toma de muestra se realizó en el domicilio particular de cada uno de los pacientes. Pero antes de aquello, se les solicitó firmar un Consentimiento Informado donde se expresa su participación voluntaria y se entrega información respecto al resguardo y la confidencialidad de los resultados ${ }^{3}$.

En primera instancia, se aplicó el Protocolo de Evaluación del Habla de González y Toledo, luego el Protocolo de Habla versus Canto para Pacientes Disártricos, el cuestionario VHI y, finalmente, la escala RASATI. Estas evaluaciones fueron aplicadas el mismo día en cada paciente con una duración de una hora aproximadamente, sin someterlos a una sesión de terapia logopédica previa ese mismo día.

\section{Resultados}

A continuación se presentan los resultados a partir de las 4 evaluaciones mencionadas con antelación y aplicadas a cada paciente con el fin de describir los resultados obtenidos.

En cuanto a los resultados de las evaluaciones que contempla este estudio, la Tabla 1 indica los puntajes del Protocolo de Habla de González y Toledo, del Protocolo de Habla versus Canto para pacientes disártricos (dividida en la subprueba Habla y en la subprueba Canto) y del VHI:

Tabla 1. Resultados globales de la muestra.

\begin{tabular}{cccccc}
\hline & $\begin{array}{c}\text { Promedio Protocolo } \\
\text { de González y } \\
\text { Toledo }\end{array}$ & $\begin{array}{c}\text { Puntaje total } \\
\text { Protocolo HvC } \\
\text { Subprueba Habla }\end{array}$ & $\begin{array}{c}\text { Puntaje total } \\
\text { Protocolo HvC } \\
\text { Subprueba Canto }\end{array}$ & Resonancia & Total VHI \\
\hline Caso 1 & 1,6 & 124 & 124 & Normal & 10 \\
\hline Caso 2 & 3,16 & 113 & 54 & Hipernasal & 30 \\
\hline
\end{tabular}

En términos generales, se puede observar que el primer participante presenta una deficiencia leve, mientras que el segundo evidencia una deficiencia moderada, según la valorización del Protocolo de Habla de González y Toledo.

El Consentimiento Informado y el proyecto de este estudio fueron previamente aprobados por el Comité de Bioética de la Universidad Adventista de Chile. 
En el puntaje promedio de este protocolo, los pacientes muestran una diferencia de 1,56 puntos a favor del segundo paciente, lo que indicaría que tiene un peor desempeño en comparación con el primero.

En cuanto a las evaluaciones realizadas a partir del protocolo creado por los investigadores de este estudio, se puede observar una diferencia mayor en la subprueba de Canto entre ambos participantes (una diferencia de 70 puntos) y ambas pruebas muestran diferencias a favor del primer participante, demostrando un mejor rendimiento en ambas modalidades. Además, el caso 1 presenta un rendimiento del $100 \%$ en habla y en canto. Como se señaló anteriormente, la segunda paciente no tiene técnica vocal y frente a estímulos rítmicos obtuvo un $43 \%$ de rendimiento, lo que genera diferencias si se compara con su desempeño en Habla (una diferencia de puntaje de 59). En cambio, el primer participante, que posee técnica vocal, no presenta diferencias importantes en la modalidad de Canto.

Si se observan los ítems evaluados en el Protocolo de Habla de González y Toledo, se puede determinar, de igual forma, que el primer evaluado tiene un mejor desempeño, a excepción de la Resonancia, donde ambos presentan una puntuación de 1. Para analizar aquello, obsérvese la Tabla 2:

Tabla 2. Resultados en Protocolo de habla y lenguaje de González y Toledo (2002).

\begin{tabular}{lcccccc}
\hline & Respiración & Fonación & Resonancia & Articulación & Prosodia & Inteligibilidad \\
\hline Caso 1 & 2 & 3 & 1 & 1 & 2 & 1 \\
\hline Caso 2 & 4 & 4 & 1 & 3 & 4 & 3 \\
\hline
\end{tabular}

Al observar la tabla 2 se puede determinar el grado de severidad de cada uno de los Procesos Motores Básicos de los pacientes. En Respiración, con una diferencia de 2 puntos entre ambos participantes, la paciente 2 presenta una deficiencia de moderada a severa, lo que se traduce en que la capacidad respiratoria está disminuida, la coordinación respiratoria y fonorespiratoria están alteradas. Además, presenta una musculatura respiratoria hipotónica y, en ocasiones, utiliza musculatura accesoria en forma de compensación. En tanto, el paciente 1 presenta una capacidad respiratoria, una coordinación respiratoria y fonorespiratoria dentro de parámetros normales, al igual que el tono muscular.

En cuanto al proceso motor básico de Articulación, el primer paciente presenta una deficiencia leve, mientras que en la segunda paciente se observa una deficiencia de moderada a severa. Se pudo observar en esta evaluación que la exactitud del movimiento en ambos se altera en la medida que aumenta la velocidad de habla; sin embargo, en la participante 2 se evidencia una mayor diferencia en este ítem de articulación. En esta paciente se observa un control motor oral de lengua y labios alterados con presencia de fasciculaciones e hipotonía, lo que dificulta la articulación de fonemas bilabiales, labiodentales, post dentales superiores, post dentales inferiores, alveolares, palatales, velares, producidos en secuencia y en palabras. En el primer participante, en cambio, existe normalidad en cuanto al control motor oral.

Dentro de la categoría de dífonos consonánticos, se observa que la paciente de sexo femenino presenta dificultad moderada en dífonos /tl/ en posición intermedia, compensaciones a través de segmentación silábica y acentuación, lo que puede ser atribuido al entrenamiento terapéutico previo. También se observa una dificultad moderada en dífonos vocálicos /ui/ y /ue/ en posición intermedia y mantiene tendencia a acentuar la última sílaba como método compensatorio terapéutico. Así mismo, en palabras polisilábicas presenta una deficiencia de moderada a severa y sigue manteniendo compensaciones. Cabe señalar que en esta paciente se observa un aumento en la severidad de la deficiencia del habla respecto a la inteligibilidad a medida que aumenta la metría de las palabras; a diferencia del paciente masculino que no presenta dificultad alguna.

En la subprueba "El Texto del Abuelo" del Protocolo de Evaluación de Habla de González y Toledo (2002), se obtuvo como resultado que el paciente con disartria leve, en un tiempo de 32 segundos, obtiene un total de 189 palabras por minuto, lo que permite analizar una velocidad de habla aumentada. Por el contrario, la paciente con disartria moderada, en un tiempo de 84 segundos, logra un total de 72 palabras por minuto, obteniendo una velocidad de habla disminuida.

En cuanto a la Prosodia, el participante 1 presenta monotonía y precipitaciones al momento de hablar; mientras que en la participante 2 se evidencia monointensidad, monotonía y ocasionalmente silencios inadecuados.

Si se observan los resultados de inteligibilidad, se puede apreciar que la participante 2 presenta una deficiencia leve al enunciar palabras, de moderada a severa al producir oraciones, severa al emitir párrafos y moderada en una conversación. Cabe señalar, que en el primer evaluado, su inteligibilidad se encuentra dentro de parámetros normales.

Otro elemento que se puede apreciar en el segundo paciente son las diadococinesias alteradas y un habla automática con una deficiencia prosódica importante. Por otra parte, el primer participante no presenta alteraciones en la prosodia.

En Fonación, la diferencia entre los individuos es de un 1 punto en la tabla general del protocolo. En el siguiente gráfico se muestra el tiempo máximo fonatorio (TMF) en relación a la producción de la /s/ y de la /a/. En él se observa que el primer paciente presenta una deficiencia moderada en la vocal sostenida /a/, lo que representa un tiempo máximo fonatorio de 10 segundos; en cambio, la segunda paciente evidencia una deficiencia moderada a severa, lo 
que en tiempo máximo fonatorio es de 5 segundos. En cuanto a producción de /s/, el caso 1 presenta una deficiencia leve, ya que logró un tiempo de 19 segundos; a diferencia del caso 2, que logra un tiempo de 9 segundos, lo que equivale a un grado de deficiencia de moderada a severa. La Figura 1 muestra el tiempo máximo de fonación según el Protocolo de Evaluación de Habla (González y Toledo, 2002):

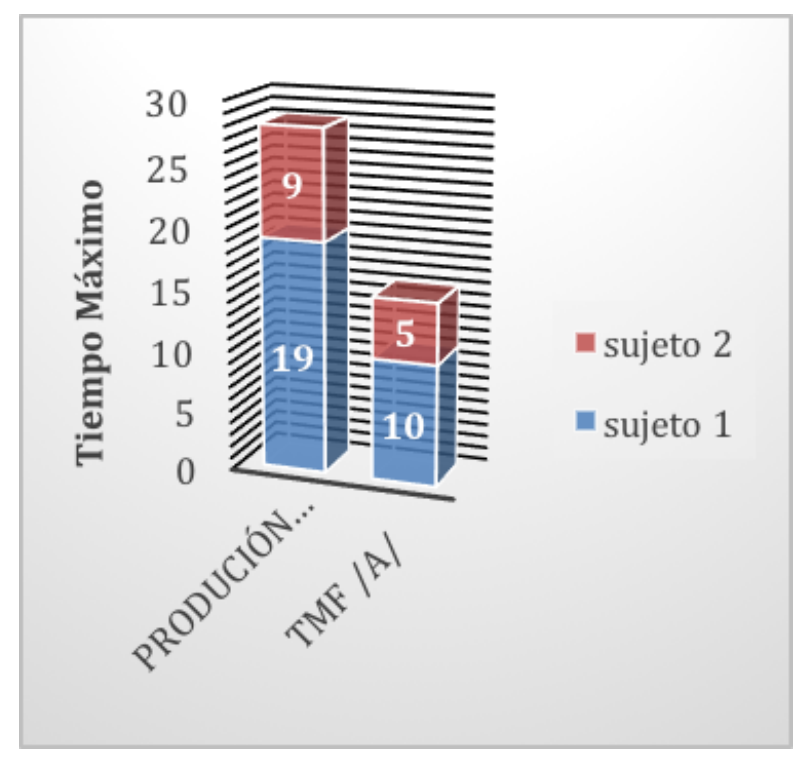

Figura 1. Tiempo Máximo en Protocolo de Evaluación de Habla de González y Toledo (2002).

En cuanto a los resultados obtenidos en el Protocolo de Habla versus Canto para pacientes disártricos, destaca la información que sigue en la Tabla 3:

Tabla 3. Ítem Habla de Protocolo Habla versus Canto para pacientes disártricos.

\begin{tabular}{lccccc}
\hline & Articulación & $\begin{array}{c}\text { Rendimiento } \\
\text { Diadococinético }\end{array}$ & Inteligibilidad & Prosodia & Fonación \\
\hline Caso 1 & 18 & 24 & 14 & 64 & 4 \\
\hline Caso 2 & 18 & 24 & 13 & 54 & 4 \\
\hline
\end{tabular}

De acuerdo a lo que indica la tabla 3, en la subprueba de Articulación no se encuentran diferencias entre los participantes. Ambos logran articular adecuadamente las palabras de corta, mediana y larga metría. De igual forma, en el rendimiento diadococinético la paciente 2 obtiene un mejor rendimiento en este protocolo en comparación con los resultados que ella misma obtuvo en el Protocolo de habla de González y Toledo (2002), ya que se utilizan facilitadores como el metrónomo y modelo del estímulo.

En la subprueba de Inteligibilidad se puede apreciar una diferencia de 1 punto a favor del caso 1. La paciente 2 , en tanto, presenta dificultad en lectura de párrafo, logrando un resultado de parcialmente logrado, pues presenta distorsión consonántica en trabantes.

En la Prosodia, se establece una diferencia de 10 puntos a favor nuevamente del caso de sexo masculino, lo que se explicaría porque la paciente 2 presenta mayor tendencia hacia las frecuencias graves, hecho que dificulta su producción en diferentes registros vocales. La misma paciente evidencia que a medida que aumenta la longitud del enunciado, disminuye la dificultad de lograr los agudos; sin embargo, el primer participante no presenta dificultad en lo anteriormente mencionado.

En cuanto a la Fonación, al momento de solicitar una /a/ sostenida, el caso 1 logra un tiempo de 10 segundos y 38 milisegundos, a diferencia de la segunda paciente, que logra un tiempo de 11 segundos.

De acuerdo a estos resultados, se puede sostener que no se observan diferencias importantes entre ambos en la subprueba de Habla del Protocolo del Habla versus Canto para pacientes disártricos. Por el contrario, en la subprueba de Canto las diferencias entre los pacientes son más evidentes. La Tabla 4 muestra los resultados obtenidos en el ítem canto en ambos pacientes: 
Tabla 4. Ítem Canto de Protocolo Habla versus Canto para pacientes disártricos.

\begin{tabular}{lcccc}
\hline & Articulación & $\begin{array}{c}\text { Rendimiento } \\
\text { diadococinético }\end{array}$ & Inteligibilidad & Fonación \\
\hline Caso 1 & 18 & 24 & 14 & 6 \\
\hline Caso 2 & 9 & 12 & 6 & 6 \\
\hline
\end{tabular}

Dentro de los resultados que se presentan, se detalla el rendimiento de la articulación en canto para estos sujetos, los cuales obtienen una diferencia de 9 puntos a favor del caso 1; debido a que, al presentar técnica vocal, logra ejecutar los estímulos de palabras de corta, mediana y larga metría sin dificultad. El caso 2, en cambio, presenta dificultades para seguir la línea melódica del estímulo; sin embargo, en el inicio de éstos la paciente logra cumplir con la tarea establecida.

En cuanto al Rendimiento Diadococinético, se puede observar que existe una diferencia de 12 puntos a favor del primer participante. Cabe destacar que, a diferencia del Protocolo de Evaluación de Habla de González y Toledo (2002), la segunda participante tiene un mejor rendimiento en las diadococinesias debido a que en esta subprueba recibe un apoyo verbal además del estímulo melódico acompañado de metrónomo, aunque no logra seguir la línea melódica.

En el ítem de Inteligibilidad se evidencia una diferencia de 8 puntos entre ambos pacientes, siendo el primer paciente el que logra un rendimiento de un $100 \%$. Por otro lado, aunque el segundo caso no logra nuevamente seguir la línea melódica en palabras, a medida que aumenta la metría del enunciado logra mantener la línea melódica del estímulo en oraciones. En el canto de párrafos, esta misma paciente, no logra seguir la línea melódica de los estímulos, ya que aumenta la dificultad y se evidencia mayor distorsión de consonantes trabantes.

Respecto de la Fonación no se observan diferencias de puntaje entre los participantes de este estudio. Sin embargo, cabe señalar que la paciente 2 logra los tonos agudos con una leve dificultad en glissando ascendente, mientras que el paciente 1 lo hace sin dificultad.

En la Figura 2 se puede observar la diferencia en segundos de ambos pacientes al producir una /a/ sostenida en habla y canto:

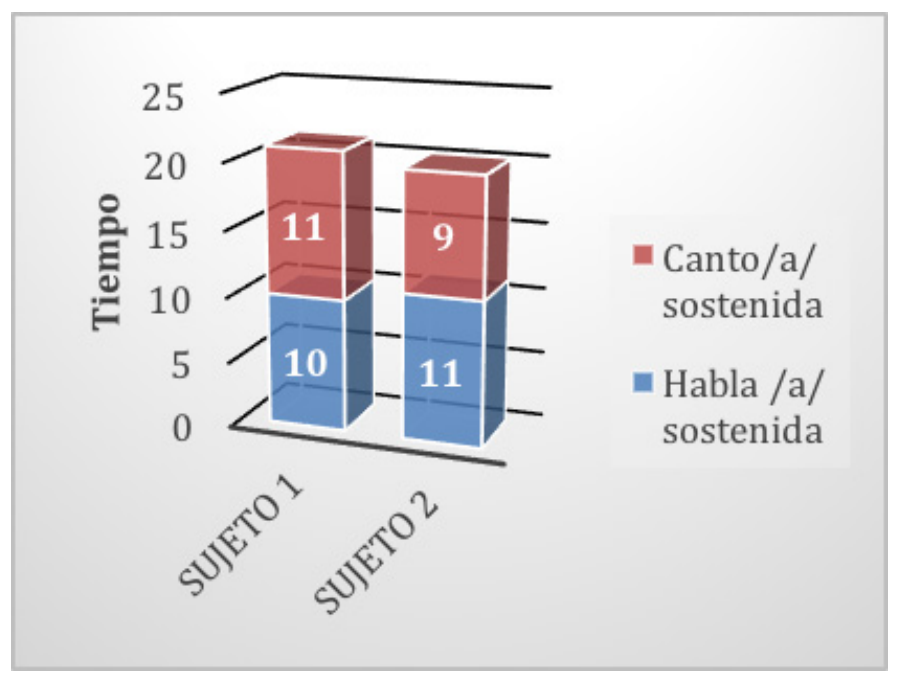

Figura 2. Tiempo en Protocolo de Habla versus Canto en pacientes disártricos.

Se puede evidenciar una diferencia entre ambos casos en habla de 1 segundo a favor de la segunda participante y en canto se aprecia una diferencia de 2 segundos a favor del primero. Cabe recordar, que este último presenta técnica vocal.

Los resultados de autopercepción de los pacientes se pueden apreciar en la siguiente Tabla 5:

Tabla 5. Resultados de Voice Handicap Index (VHI 30)

\begin{tabular}{lccc}
\hline & Funcional & Físico & Emocional \\
\hline Caso 1 & 4 & 6 & 0 \\
\hline Caso 2 & 17 & 13 & 0 \\
\hline
\end{tabular}


Se observa que tanto en la discapacidad funcional y física de la voz se presentan diferencias de puntaje de 13 y 7 puntos respectivamente entre ambos participantes a favor de la segunda paciente, lo que significa que ésta presenta una discapacidad moderada en cuanto a su autopercepción vocal. Sin embargo, esta evaluación se vio sesgada ya que la paciente no logró diferenciar si sus dificultades vocales estaban provocadas por su voz propiamente tal o por su alteración articulatoria.

En cuanto a los resultados de RASATI, observe la siguiente Tabla 6:

Tabla 6. Calidad de voz según RASATI.

\begin{tabular}{ccccccc}
\hline & Ronquera & Aspereza & Soplocidad & Astenia & Tensión & Irregularidad \\
\hline Caso 1 & 1 & 2 & 0 & 0 & 1 & 0 \\
\hline Caso 2 & 0 & 1 & 1 & 0 & 3 & 0 \\
\hline
\end{tabular}

Según RASATI, el primer participante presenta una calidad de voz predominantemente áspera, acompañada de una ronquera y tensión leve; a diferencia de la segunda, que presenta una calidad de voz predominantemente tensa, acompañada de aspereza y soplocidad leve.

Cabe destacar que los resultados que arrojó el RASATI se obtuvieron mediante el juicio de expertos con aproximadamente entre 5 y 7 años de experiencia.

\section{Discusión y conclusiones}

El objetivo del presente estudio fue describir y caracterizar el rendimiento del habla y del canto en dos pacientes con accidente cerebrovascular cerebeloso con antecedentes anamnésicos distintos por medio de los instrumentos mencionados con anterioridad.

En relación a la evaluación realizada mediante el Protocolo de González y Toledo (2002), en la categoría de respiración, ambos personas presentan alteración; el primer paciente presenta una deficiencia leve, evidenciada en una menor capacidad respiratoria, coordinación respiratoria y coordinación fonorespiratoria. La segunda, presenta una alteración más notoria en estos mismos procesos. En base a lo mencionado anteriormente, se puede destacar que lo evaluado en esta categoría coincide con lo que plantea la Guía de Intervención Logopédica en la Disartria (Melle, 2008), la cual manifiesta que los pacientes con disartria atáxica en diferentes grados de severidad podrían también presentar alteraciones en la respiración. Esta guía también plantea que este tipo de pacientes presentan una insuficiencia fonatoria, lo que fue evidente al momento de evaluar la fonación por medio de la producción de la vocal /a/ de forma sostenida. Tanto el primero como la segunda paciente presentan una deficiencia, moderada y moderada $a$ severa respectivamente, en esta categoría.

Según los valores obtenidos en la categoría de articulación, la alteración más notoria la presenta el caso 2; sin embargo, en ambos pacientes se observan características de habla escándida, lo que coincide con lo expuesto por Melle (2008).

En base a los resultados obtenidos en el ítem de Prosodia, la Guía de Intervención Logopédica en la Disartria (Melle, 2008) refieren los mismos hallazgos de este estudio, los cuales son acentuación excesiva, pausas entre palabras, exceso prosódico y lentitud en la tasa de habla y acentuación excesiva en sílabas. Es relevante mencionar que los resultados que arrojó el Protocolo de Habla versus Canto para pacientes disártricos (de autoría propia) coincide con la bibliografía expuesta, por lo cual puede considerarse un aporte al momento de evaluar estas habilidades en este tipo de pacientes.

En relación a la evaluación perceptual de la calidad de la voz según RASATI, los resultados obtenidos en un estudio realizado sobre disartrias de González y Bevilacqua (2012) y los de este estudio de caso no son del todo concordantes. Lo anterior se debe a que, según los autores, los pacientes con disartria atáxica deben presentar una calidad de voz normal - ronca; mientras que en este estudio el participante 1 presenta una calidad de voz predominantemente áspera, acompañada en menor medida de ronquera y tensión leve y la segunda participante presenta una calidad de voz predominantemente tensa, acompañada de aspereza y soplocidad leve.

De acuerdo a los hallazgos de este estudio se evidencia un mayor compromiso de los procesos motores básicos del habla y de los parámetros funcionales de la misma en la segunda paciente, equivalente a la severidad del cuadro. Lo anterior se explica por los datos clínicos y evolución de la patología.

Si se revisa la prevención y promoción del ACV en Chile, se sabe que las campañas y políticas públicas apuntan a las patologías médicas de base que generan riesgo cardiovascular como la hipertensión arterial. Por el contrario, poco se educa respecto a los factores protectores de la rehabilitación, como ocurre en el caso del entrenamiento físico, la 
escolaridad, el entrenamiento musical, la alimentación o los hábitos saludables, entre otros factores, los cuales permiten una curva de rehabilitación más ascendente luego de padecer de un ACV. En base a esto, los pacientes caracterizados logran diferentes rendimientos debido a todas las diferencias expuestas, lo que genera un impacto diferente al momento de reinsertarse en el contexto laboral, familiar y social.

Finalmente, se observa que un sujeto con entrenamiento musical previo a un accidente cerebro vascular genera una base de procesos motores básicos entrenados con un mayor nivel de complejidad, que le permiten poseer mayor especificidad y entrenamiento asociadas a estas habilidades, lo que se traduce en una capacidad respiratoria pre mórbida mayor, una mejor coordinación respiratoria y fonorespiratoria, la presencia de entrenamiento de técnica vocal adecuada, unas cuerdas vocales más resistentes y elásticas, una mayor fuerza de aducción cordal (Uzcanga, Fernández, Maquéz, Sarrasqueta y García-Tapia, 2006), mejores tiempos máximos de fonación y espiración, una regulación más eficiente y adecuada de la presión subglótica, órganos fonoarticulatorios (labios, lengua, mejillas, velo del paladar, etc.) ejercitados para realizar movimientos más precisos, más rápidos, más coordinados y exactos y una mayor proyección de la voz y del foco resonancial que potencian la habilidades comunicativas previas a un daño neurológico. No obstante, a partir de este estudio, no se puede concluir que la técnica vocal es el único factor protector que contribuya al éxito terapéutico en pacientes con ACV cerebeloso. Esto se debe a que los casos aquí expuestos poseen diferencias clínicas, además del entrenamiento vocal, muy distintas. Sin embargo, incluir este entrenamiento puede ser beneficioso para la eficiencia y eficacia de la terapia, lo que puede ser dilucidado por estudios posteriores que consideren una muestra más homogénea en cuanto a sus características clínicas y personales. Es pues, considerada una limitación la situación basal de la muestra debido a que no comparten la misma severidad en el cuadro disártrico, entre otras diferencias mencionadas con anterioridad. Por lo cual, próximas investigaciones debiesen incluir, además de datos asociados a patrones espirométricos o estudios de función pulmonar que puedan influir en resultados en la evaluación del habla y del canto en este tipo de pacientes, mediciones electroglotográficas y aerodinámicas para un análisis de resultados mayormente objetivos.

\section{Referencias bibliográficas}

Custodio, N. y Cano - Campos, M. (2017). Efectos de la música sobre las funciones cognitivas. Revista Neuropsiquiatría, 61 - 71. DOI: $10.20453 /$ rnp.v80i1.3060

Duffy, J. (2005). Motor speech disorders: substrates, differential diagnosis and management. Second edition. St. Louis, MO: Elsevier Mosby.

Ferri, L. (2015). Cerebelo y lenguaje: intervención logopédica en sus trastornos. Revista de Neurología , 57-62. DOI: 10.33588/ rn.60S01.2015020

González, R. y Bevilacqua, J. (2012). Las Disartrias. Revista Hospital Clínico Universidad de Chile, 23, $299-309$.

González, R. y Toledo, L. (2002). Protocolo de Evaluación de Habla. Documento no publicado.

Hirano, M. (1981). Clinical examination of voice. Disorders of human communication, 5, 1-99.

Kent, R., Anley, J., Duffy, J., Thomas, J., Welsmer, G., y Stuntebeck, S. (2000). Ataxic Dysarthria. Journal of Speech, Language and Hearing Research, 43, 2175-1289.

Melle, N. (2008). Guía de intervención logopédica en la disartria. Málaga, España: Síntesis.

Ministerio de Salud. (2015). Guía clínica AUGE Accidente Cerebro Vascular Isquémico. En M. De Salud, Guía clínica AUGE Accidente Cerebro Vascular Isquémico (pp. 1-130). Santiago, Chile.

Ministerio de Salud. (2011). Citado en Plan de acción ataque cerebrovascular. Acciones estratégicas para el enfrentamiento del ACV en Chile 2014-2020. Recuperado en https://www.minsal.cl/sites/default/files/Minuta-Ataque-Cerebro-Vascular-28-10-2014.pdf

Montalvo, J. y Moreira-Vera, D. (2016). El cerebro y la música. Revista Ecuatoriana de Neurología, 25, 50-55.

Núñez-Batalla, F., Corte-Santos, P., Señaris-González, B., Llorente-Pendás, J. L., Górriz-Gil, C., y Suárez-Nieto, C. (2007). Adaptación y validación del índice de incapacidad vocal (VHI-30) y su versión abreviada (VHI-10) al español. Acta Otorrinolaringológica Española, 58(9), 386-392. DOI: 10.1016/s0001-6519(07)74954-3

Pinho, S. M. R. y Pontes, P. (2002). Escala de avaliação perceptiva da fonte glótica: RASAT. Vox Brasilis, 3(1), 11-3.

Ropper, A., Brown, R., y Klein, J. (2016). Principios de neurología. Madrid, España: Mc GrawHilll.

Soria - Urios, G., Duque, P. y García - Moreno, J. (2011). Música y cerebro (II): evidencias cerebrales del entrenamiento musical. Revista de Neurología, 739-74. DOI: 10.33588/rn.5312.2011475

Urban, P. (2013). Speech motor deficits in cerebellar infarctions . Brain \& Language, 323-326. DOI: 10.1016/j.bandl.2013.10.001

Uzcanga, M., Fernández, S., Maquéz, M., Sarrasqueta, L. y García-Tapia, R. (2006). Voz cantada. Revista Médica Universitaria Navarra 50(3) 2, 49-55. 